\title{
A EPISTEMOLOGIA DE THOMAS KUHN: UM OLHAR PARA A MATEMÁTICA
}

\section{Paola dos Santos Aquino1; Juliana Gabriele Kiefer 2; Marcos Alexandre Alves³}

\section{RESUMO:}

Com base na obra A Estrutura das Revoluções Científicas e artigos científicos apresentamos alguns conceitos da teoria de Thomas Kuhn, destacando o conceito de paradigma. O objetivo deste artigo é realizar uma pesquisa qualitativa bibliográfica com vistas a abordar a epistemologia de Thomas Kuhn no que tange aos paradigmas tecendo aproximações com a Matemática. Para tanto, o corpus deste artigo é formado por apenas três produções acadêmicas obtidas através de levantamentos na Biblioteca Digital Brasileira de Teses e Dissertações - BDTD. A partir dos trabalhos identificados conclui-se que a teoria kuhniana pode ser aplicada em seus aspectos centrais ao estudo histórico e epistemológico da matemática.

Palavras-chave: Ciência; Mapeamento; Paradigmas matemáticos.

Eixo Temático: Educação, Cultura e Comunicação (ECC).

\section{INTRODUÇÃO}

O presente artigo é decorrente de reflexões realizadas na disciplina de Fundamentos Epistemológicos da Ciência, disciplina obrigatória no Curso de Doutorado em Ensino de Ciências e Matemática da Universidade Franciscana. Nesta disciplina foram realizadas discussões acerca da Epistemologia e ensino de ciências; Epistemologia da complexidade e paradigma emergente (Bauman, Morin e Souza Santos); Epistemologias contemporâneas e o ensino de ciências (Popper, Kuhn, Lakatos, Bachelard, Feyerabend); Epistemologia hermenêutica e educação (Schleiermacher e Gadamer); e também, Epistemologia, Técnica e Ética científica (Jonas e Morin).

De modo mais específico, pretende-se, neste artigo, realizar uma pesquisa

\footnotetext{
${ }^{1}$ Doutoranda no Programa de Pós-graduação em Ensino de Ciências e Matemática - Universidade Franciscana - UFN. Bolsista da Coordenação de Aperfeiçoamento de Pessoal do Ensino Superior (CAPES). E-mail: paolasantosmtm@gmail.com

${ }^{2}$ Doutoranda no Programa de Pós-graduação em Ensino de Ciências e Matemática - Universidade Franciscana - UFN. Bolsista da Coordenação de Aperfeiçoamento de Pessoal do Ensino Superior (CAPES). E-mail: juliana_kiefer@hotmail.com

${ }^{3}$ Docente no Programa de Pós-graduação em Ensino de Ciências e Matemática - Universidade Franciscana - UFN. E-mail: maralexalves@gmail.com
} 
qualitativa bibliográfica com vistas a abordar a epistemologia de Thomas Kuhn no que tange aos paradigmas tecendo aproximações com a Matemática.

Thomas Samuel Kuhn (1922-1996) nasceu em Cincinnati, Ohio, Estados Unidos, no dia 18 de julho de 1922 e faleceu em Cambridge, Massachusetts, Estados Unidos, no dia 17 de junho de 1996. Realizou o curso de física (1943) na Universidade de Harvard, onde também recebeu o título de mestre (1946) e doutor (1949), ambos na área de Física.

Além de físico, é considerado um grande filósofo da ciência estadunidense, seu trabalho incidiu sobre história e filosofia da ciência tornando-se um marco no estudo do processo de construção de fatos científicos. Seus trabalhos acadêmicos resultaram em alguns livros, a saber: A Revolução Copernicana (1957), Estruturas da Revolução Científica (1962), A tensão essencial (1977), Teoria do Corpo Negro e Descontinuidade Quântica (1979) e O caminho desde a estrutura (2000).

Com a publicação do livro Estrutura das Revoluções Científicas em 1962, foi que Kuhn se tornou conhecido não mais como físico, mas, sim, como intelectual voltado para a história e a filosofia da ciência. O livro foi reeditado em 1970 com algumas observações adicionais, principalmente sobre o conceito de paradigma.

$\mathrm{Na}$ seção seguinte apresentam-se brevemente considerações sobre este conceito de acordo com as ideias defendidas por Kuhn, bem como de outros conceitos principais de sua teoria como, por exemplo, ciência normal, revolução científica e incomensurabilidade.

\subsection{UM POUCO SOBRE A EPISTEMOLOGIA DE THOMAS KUHN}

Thomas Kuhn foi um físico e estudioso que se dedicou à área da filosofia da ciência contemporânea. Com suas ideias precursoras para o desenvolvimento científico, a epistemologia de Kuhn compreende que a produção do conhecimento científico tem por princípio a observação não neutra, não indutiva, de caráter construtivo, criativo e não definitivo.

$\mathrm{Na}$ teoria de Kuhn a ciência pode ser descrita por meio de um modelo de desenvolvimento. A figura 1 apresenta três modelos de ciência Kuhniano. 


\section{QUFN}

Figura 1. Modelos de Ciência Kuhniano

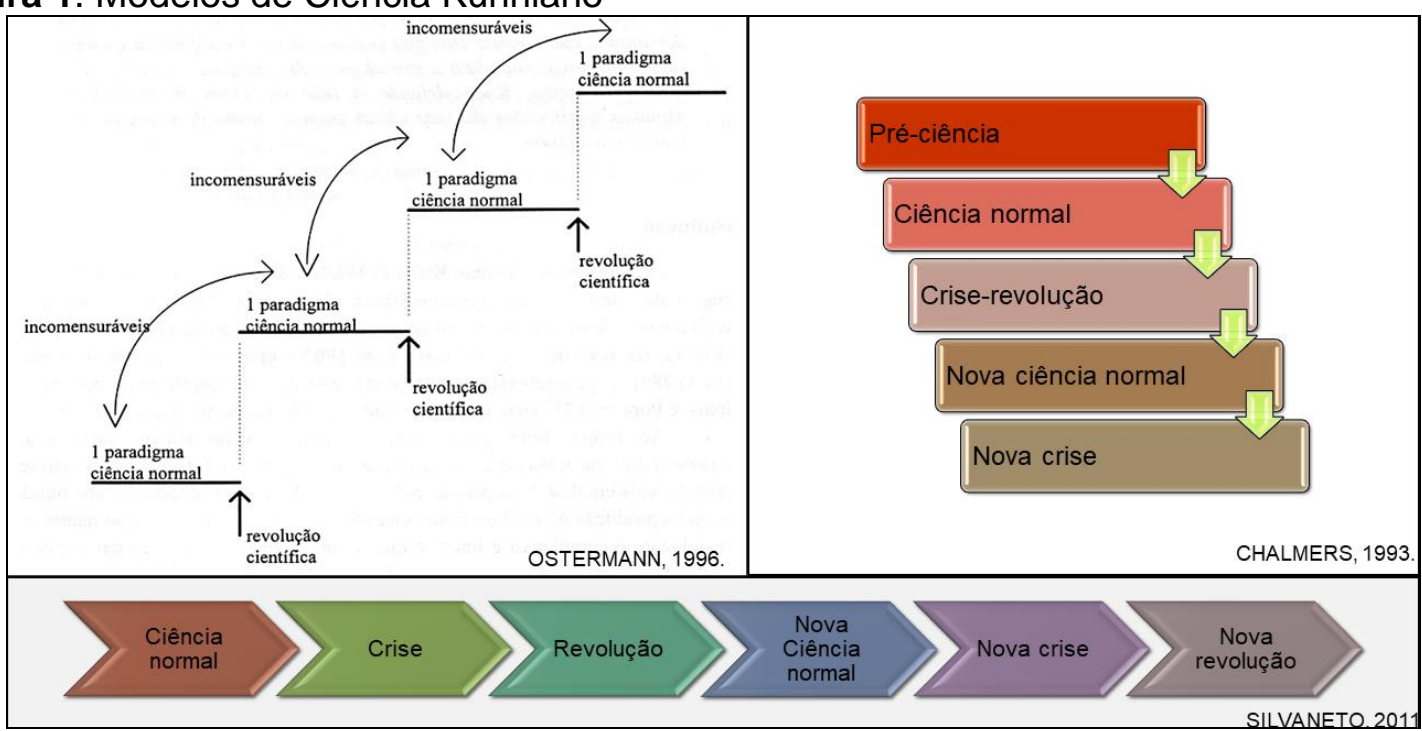

Fonte: elaborada pelos autores conforme Ostermann (1996), Chalmers (1993) e Silva Neto (2011)

O modelo proposto por Ostermann (1996) destaca uma sequência de períodos de ciência normal em que a comunidade científica de pesquisadores adere a um paradigma que são interrompidas por revoluções científicas. Nas revoluções científicas ao depararem-se com anomalias no paradigma resultam em rupturas.

Chalmers (1993) descreve inicialmente uma atividade desorganizada e diversa (pré-ciência) que antecede a formação da ciência estruturada pela comunidade científica ligada a um paradigma. Os cientistas que trabalham dentro de um paradigma praticam a ciência normal que ao desenvolver e articular o paradigma deparam-se com falsificações, nestas dificuldades surgem as crises que são resolvidas por um novo paradigma, esta fase é denominada revolução científica. Este novo paradigma direciona a atividade científica normal até que encontre dificuldades e ocasione uma nova revolução.

Silva Neto (2011) explica em seu modelo de ciência, que quando uma comunidade científica estabelece um paradigma para explicar e resolver problemas cotidianos de uma ciência, neste processo ao responder aos enigmas e fundando a ciência normal. Os paradigmas propiciam meios para resolver os quebra cabeças, mas ao encontrarem limitações ocorrem as crises e diante destas anomalias surgem novos paradigmas e reinicia o processo de migrar de um paradigma ao novo é a revolução científica. 
Os modelos destacam que a ciência proposta por Kuhn não é linear ou contínua mas se dá por saltos ou revoluções. De acordo com Ostermann (1996):

- modelo kuhniano encara o desenvolvimento científico como uma sequência de períodos de ciência normal, nos quais a comunidade científica adere a um paradigma. Estes períodos, por sua vez, são interrompidos por revoluções científicas, marcadas por crises/anomalias no paradigma dominante, culminando com sua ruptura. A crise é superada quando surge um novo candidato a paradigma. Ao comparar o antigo e o novo paradigma, Kuhn defende a tese da incomensurabilidade (OSTERMANN, 1996, p. 184).

A epistemologia proposta por Kuhn apresenta alguns conceitos principais que orientam sua teoria conforme descritos na Figura 2.

Figura 2. Conceitos Principais de Kuhn.

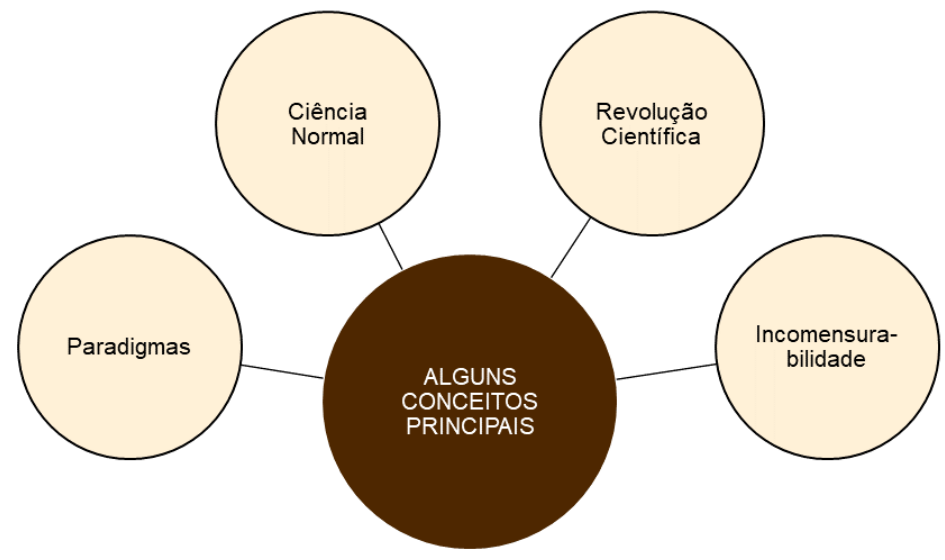

Fonte: elaborada pelos autores.

A princípio, discorremos sobre os conceitos de ciência normal, paradigma, ciência revolucionária e incomensurabilidade já mencionados na figura 2 acerca destas convicções principais para compreender o pensamento Kuhniano. Segundo Kuhn (1989), o progresso da ciência ocorre por meio da sucessão de paradigmas, que são fundamentais à ciência.

O paradigma é o conceito fundamental desta teoria, Kuhn (1989, p. 13) considera este termo como "[...] as realizações científicas universalmente reconhecidas que, durante algum tempo, fornecem problemas e soluções modelares para uma comunidade de praticantes de uma ciência”. Ou seja, a teoria Kuhniana se refere ao paradigma como um modelo ou padrão de saberes que propiciam realizar as pesquisas científicas por uma determinada comunidade científica. 
Segundo Ostermann (1996), o paradigma possui dois sentidos, um geral e outro restrito. O sentido geral consiste na designação de todos os compromissos de pesquisa de uma comunidade científica que ordena todos os detalhes das práticas de pesquisa. E o sentido restrito se refere aos "limites preestabelecidos e relativamente inflexíveis fornecidos pelo paradigma, ou seja, modelar a solução de novos problemas segundo problemas exemplares" (p. 188).

Marín (2007) apresenta a discussão da palavra paradigma destacando a definição de Kuhn que diz "o que os membros de uma comunidade científica compartilham e, reciprocamente, uma comunidade científica consiste em homens que compartilham um paradigma" (p. 36). O autor além de definir e identificar este termo da teoria de Kuhn identifica algumas características conforme é mostrada na figura 3.

Figura 3. Características do Paradigma.

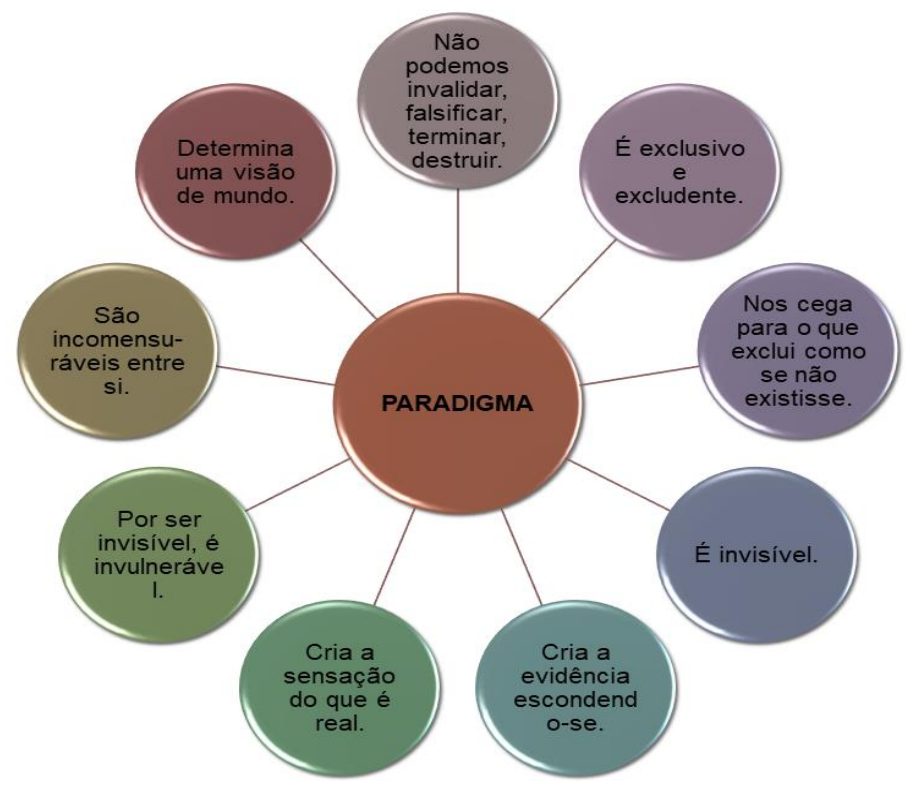

Fonte: elaborada pelos autores conforme Marín (2007).

Conforme Marín (2007) estes conjuntos de valores e conhecimentos que pertencem a uma comunidade, são os paradigmas, que não desaparecem, apenas encontram limitações.

Estas limitações propiciam a percepção das falhas nos quebra cabeças que Kuhn conceitua de anomalias do paradigma, ou seja, "o reconhecimento de que, de 
alguma maneira, a natureza violou as expectativas paradigmáticas que governam a ciência normal" (1989, p. 78).

O paradigma está relacionado à ciência normal. Conforme Kuhn (1989) a ciência normal possui suas determinações, mas não é necessariamente definida por regras. Esta relação da ciência normal ao paradigma é destacada por Kuhn (1989, p. 45), do seguinte modo:

\begin{abstract}
A ciência normal não tem como objetivo trazer à tona novas espécies de fenômeno; na verdade, aqueles que não se ajustam aos limites do paradigma frequentemente nem são vistos. Os cientistas também não estão constantemente procurando inventar novas teorias; frequentemente mostram-se intolerantes com aquelas inventadas por outros. Em vez disso, a pesquisa científica normal está dirigida para a articulação daqueles fenômenos e teorias já fornecidos pelo paradigma (KUHN,1989, p. 45).
\end{abstract}

A ciência normal analisa os fenômenos e as teorias oriundas do paradigma. A ciência normal determina a visão do cientista devido às pequenas áreas investigadas. As restrições fundamentadas no paradigma possibilitam 0 desenvolvimento científico ( OSTERMANN, 1996).

Uma analogia é estabelecida entre a ciência normal e quebra-cabeça, devido este conceito possibilitar a previsibilidade e segurança para a resolução de problemas estabelecidos pelo paradigma. Conforme Kuhn (1989, p. 60) "[...] uma comunidade científica, ao adquirir um paradigma, adquire igualmente um critério para a escolha de problemas que, enquanto o paradigma for aceito, poderemos considerar como dotados de uma solução possível".

Deste modo, a ciência normal se depara com percalços que limitam as suas soluções e explicações dos problemas. Neste momento, de acordo com Ostermann (1996, p. 190) "os problemas, ao invés de serem encarados como quebra-cabeças, passam a ser considerados como anomalias, gerando um estado de crise", este período de limitações é denominado de ciência extraordinária.

Os paradigmas não são rejeitados pelos cientistas apenas por se depararem com as anomalias. A teoria após se tornar um paradigma somente terá suas limitações quando não houver outra possibilidade. $E$ esta transição de um antigo paradigma ao novo é caracterizado por Kuhn (1989) como revolução científica. 
Neste período de transição do antigo ao novo paradigma, as novas teorias que resolverão as anomalias juntamente com as novas teorias permitirão predições diversas da precursora. $E$ assim por definição de Kuhn a expressão de incomensurabilidade de paradigmas é empregado (OSTERMANN, 1996).

\section{ASPECTOS METODOLÓGICOS}

A abordagem metodológica desta investigação é qualitativa de cunho bibliográfico, pois é elaborada a partir de material já publicado, como publicações em artigos científicos, dissertações e teses "[...] com o objetivo de colocar o pesquisador em contato direto com todo material já escrito sobre o assunto da pesquisa." (PRODANOV; FREITAS, 2013, p. 54). Nesse sentido, Ferreira (2002, p. 258) afirma que as pesquisas bibliográficas objetivam: [...] responder que aspectos e dimensões vêm sendo destacados e privilegiados em diferentes épocas e lugares, [...]". Assim, pretende-se, neste artigo, apresentar o que já existe sobre a temática das revoluções científicas de Thomas Kuhn considerando aproximações com a Matemática.

O levantamento bibliográfico foi realizado por meio do repositório da Biblioteca digital brasileira de teses e dissertações (BDTD) no mês de julho de 2021. Foram utilizados os seguintes termos de busca e obtidos os referidos quantitativos: "paradigma"(título); "matemática" (título) (8 pesquisas), "paradigma"(título); "matemática" (todos os campos) (18 pesquisas); "paradigma"(resumo); "matemática" (resumo); "Kuhn” (resumo) (32 pesquisas).

A partir disso, foi realizada a leitura dos resumos das investigações e adotados os seguintes critérios: 1) abordar as ideias de Kuhn; 2) e também estar relacionadas com a matemática e/ou ensino de matemática. Assim, o corpus de investigações ficou constituído por 3 trabalhos (QUADRO 1), sendo uma dissertação de mestrado $(M)$ e duas teses de doutorado (D). 
EDUCAÇÃO, SAÚDE

ETECNOLOGIA

26 A 28 DE OUTUBRO DE 2021

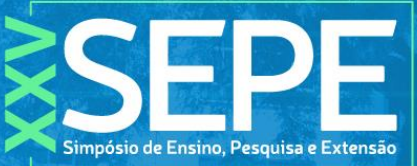

QUFN

Quadro 1: Corpus de análise

\begin{tabular}{|c|c|c|c|c|}
\hline Ano & Autor & M ou D & IES & Título \\
\hline 2005 & $\begin{array}{c}\text { Martins, João } \\
\text { Carlos Gilli }\end{array}$ & D & $\begin{array}{c}\text { Universidade } \\
\text { Estadual Paulista }\end{array}$ & Sobre revoluções científicas na matemática \\
\hline $\begin{array}{c}\text { Santos, } \\
\text { Eberth } \\
\text { Eleuterio dos }\end{array}$ & D & $\begin{array}{c}\text { Universidade } \\
\text { Estadual de } \\
\text { Campinas }\end{array}$ & $\begin{array}{c}\text { O infinito de George Cantor: uma revolução } \\
\text { paradigmática no desenvolvimento da } \\
\text { matemática }\end{array}$ \\
\hline Lorin, João \\
Henrique
\end{tabular}

Fonte: Organizado pelos autores a partir da BDTD.

A partir do Quadro 1, observa-se que as pesquisas estão relacionadas de modo específico com a matemática, sendo abordadas as revoluções científicas na matemática no que tange ao estudo da álgebra (MARTINS, 2005), infinito de George Cantor (SANTOS, 2008) e do paradigma pitagórico ao paradigma euclidiano (LORIN, 2009).

Com o corpus já selecionado passou-se a buscar nele exemplos de relações entre a epistemologia de Thomas Kuhn e a matemática. Tais elementos serão expostos nos tópicos que veremos a seguir.

\section{PARADIGMAS NA MATEMÁTICA: ALGUNS EXEMPLOS}

O primeiro trabalho identificado foi a tese de João Carlos Gilli Martins, defendida no ano de 2005 na Universidade Estadual Paulista. O autor constatou unanimidade entre os filósofos da Matemática a compreensão de que as revoluções científicas, na forma como são apresentadas em $A$ Estrutura das Revoluções Científicas, de Thomas S. Kuhn, não ocorrem na Matemática.

Entretanto, fundado no Modelo Teórico dos Campos Semânticos e tendo a história da Matemática como cenário mais especificamente, a história da Álgebra, seu objetivo foi mostrar o contrário, ou seja:

[...] Mostrar que a obra Kitab al mukhtasar fi hisab al-jabr wa I-muqabalah, de al-Khwarizmi, inaugura o primeiro período de pesquisa normal no desenvolvimento da Álgebra na Europa, um período altamente cumulativo e 
extraordinariamente bem sucedido em seus objetivos paradigmáticos e que se estendeu até as décadas iniciais do século XIX.

[...] Mostrar, ainda, que a demonstração do, hoje denominado, Teorema Fundamental da Álgebra, por Gauss, e a publicação do trabalho Sobre a resolução algébrica de equações, de Abel, trouxe à luz, na forma de um fato, uma anomalia irresolúvel do primeiro paradigma da Álgebra no Velho Continente. A partir daí, abriu-se um período de pesquisa extraordinária no âmbito dessa disciplina um período revolucionário de onde viria emergir um novo período de pesquisa normal, um novo paradigma para a Álgebra os sistemas algébricos abstratos fundado nas realizações matemáticas de Galois, Peacock e Hamilton (MARTINS, 2005, resumo).

A partir do seu estudo, o pesquisador conclui que "[...].o processo pelo qual o conhecimento algébrico foi construído pode ser analisado pela ótica de $A$ Estrutura das Revoluções Científicas." (MARTINS, 2005, p. 175). Na Figura 4 apresenta-se, com base em suas conclusões, um esquema relacionando os aspectos de Kuhn ao conhecimento algébrico.

Figura 4. Paradigma da álgebra aritmética para o paradigma da álgebra abstrata.
Realizações científicas estruturadas por um paradigma e
norteadoras do período de pesquisa normal:
As álgebras de al- Khwarizmi e de al-Karaji;
Período de pesquisa normal:
O desenvolvimento da Álgebra, nos seis séculos que separaram
Fibonacci de Gauss e de Abel, foi contínuo e altamente cumulativo;
Emergência
de anomalias:
- A demonstração do Teorema Fundamental da Álgebra garantindo que toda equação algébrica de grau $n>0$ a coeficientes complexos admitia pelo menos uma raiz complexa;
- A demonstração de que as equações de grau maiores do que quatro não eram resolúveis pelo método estabelecido pela pesquisa normal.
a conjunção desses dois fatos punha em evidência que o velho paradigma não era capaz de solucionar os problemas que ele próprio estabelecera como importantes.

Estabelecida a crise revolucionária, abre-se o período de pesquisa extraordinária e, com ela, as inquietações $e$ as indefinições. No entanto, se nesse período de crise a percepção da anomalia é muito mais evidente, essa "percepção [...] desempenhou um papel essencial na preparação do caminho que permitiu a percepção da novidade" (Kuhn; 1982, p. 84)
Tanto Galois com a sua teoria, quanto Peacock com o seu tratado sobre álgebra, vistos de hoje, expressavam mais do que a percepção - a emergência do novo paradigma da Álgebra, um paradigma estruturado na forma de um sistema algébrico abstrato.

Fonte: elaborada pelos autores conforme Martins (2005).

O autor ressalta que tinha como primeiro objetivo de seu trabalho "[...] analisar o desenvolvimento das três grandes áreas da Matemática: a Álgebra, a Geometria e a Análise sob a ótica da teoria da ciência de Thomas S. Kuhn" (MARTINS, 2005,p. 173). Entretanto, "[...] com o andar da carruagem essa tarefa mostrou-se hercúlea, fora de propósito para uma pesquisa marcada pela delimitação do tempo" (MARTINS, 2005,p. 173), sendo assim focou no estudo da álgebra. 
A partir do corpus de análise da nossa investigação, observamos que os dois próximos trabalhos identificados buscaram explorar áreas diferentes das que Martins (2005) analisou. O segundo trabalho identificado também foi uma tese, defendida em 2008 na Universidade Estadual de Campinas por Eberth Eleutério dos Santos.

O principal objetivo de em sua tese foi:

argumentar que o núcleo dos resultados matemáticos obtidos por Georg Cantor no final do século XIX, cujas ideias mais fundamentais orbitam em torno de sua especial concepção de infinito, possui as necessárias qualidades de inovação e grandeza para ser considerado como um avanço revolucionário em matemática (SANTOS, 2008, p. 15).

O pesquisador concluiu, motivado pelas ideias de Thomas Kuhn, que o aparecimento da Teoria de Conjuntos de Cantor representa a revisão de um antigo paradigma filosófico-matemático, sendo que este paradigma teve sua primeira elaboração lógica e filosófica com Aristóteles e se desenvolveu como a maneira dominante de pensar a ideia de infinito (SANTOS, 2008). Assim, a partir de seu estudo, foi possível vislumbrar que o desenvolvimento da matemática também pode ser lido através do enfoque das revoluções, e que um exemplo disto é representado pelo esforço intelectual de Cantor.

Cabe ressaltar ainda que Santos (2008, p.189) traz outros dois exemplos de eventos revolucionários na história da matemática, sendo eles:

[...] a teoria das proporções dos pitagóricos, totalmente revisada após o golpe da descoberta dos incomensuráveis, através dos excelentes trabalhos de geometrização de Teeteto e Eudoxo, culminando, finalmente, no enorme trabalho de fundamentação da geometria por Euclides (Lintz 2007).

[...] advento do cálculo diferencial que proveu a matemática com novos conceitos e recursos tais que não se pode dizer que ali não houve um fantástico salto qualitativo no desenvolvimento da matemática ocidental (SANTOS, 2008, p.189, grifo nosso)

A terceira pesquisa é uma dissertação de mestrado, defendida em 2009 por João Henrique Lorin na Universidade Estadual de Maringá que teve como objeto de estudo a análise da constituição das medidas incomensuráveis - ou números irracionais - pela teoria da ciência elaborada por Thomas Kuhn. Sendo assim o problema de pesquisa foi delimitado na compreensão da constituição do paradigma pitagórico e do paradigma euclidiano. 
O principal objetivo de seu trabalho foi identificar se a descoberta das medidas incomensuráveis causou ruptura no desenvolvimento da ciência matemática, identificando na elaboração histórica desta teoria uma descontinuidade no processo de construção do conhecimento matemático. $O$ autor conclui que a teoria kuhniana pode ser aplicada em seus aspectos centrais ao estudo histórico e epistemológico da matemática, em específico, na mudança da matemática pitagórica para a geometria euclidiana.

\section{CONSIDERAÇÕES FINAIS}

Este trabalho teve como finalidade realizar uma pesquisa qualitativa bibliográfica com vistas a abordar a epistemologia de Thomas Kuhn no que tange aos paradigmas tecendo aproximações com a Matemática.

A partir do estudo realizado neste artigo concluímos que ainda são pouco expressivas, em termos quantitativos, as pesquisas que possuem como objeto de investigação as revoluções científicas na concepção de Thomas Kuhn, no contexto da matemática. Além disso, a partir dos exemplos aqui apresentados, por meio das pesquisas de Martins (2005), Santos (2008) e Lorin (2009) conclui-se que a teoria kuhniana pode ser aplicada em seus aspectos centrais ao estudo histórico e epistemológico da matemática.

Por fim, ressalta-se a importância do estudo de Kuhn para a ciência de modo geral, mas buscou-se verificar de modo específico relações com a matemática, tendo em vista que as primeiras autoras deste artigo, ambas possuem formação na área de matemática. Deste modo, com os resultados obtidos observamos que os conhecimentos matemáticos podem ser articulados sob a ótica da teoria kuhniana.

Pretende-se ampliar a investigação realizada aqui tomando como base outros repositórios de busca como por exemplo, em anais de eventos e periódicos da área.

\section{AGRADECIMENTOS}

A Coordenação de Aperfeiçoamento de Pessoal do Ensino Superior (CAPES). 


\section{QUFN}

\section{REFERÊNCIAS}

CHALMERS, A.F. (1993). O que é Ciência, afinal? São Paulo: Brasiliense.

KUHN, T. S. A estrutura das revoluções científicas. São Paulo: Perspectiva, 1989.

LORIN, J. H. Uma revolução científica na matemática: do paradigma pitagórico ao paradigma euclidiano. Maringá, 2009. Dissertação de Mestrado- Universidade Estadual de Maringá, 2009.

MARIN, L. F. La noción de paradigma. Signo pensam., Bogotá, n. 50, p. 34-45,

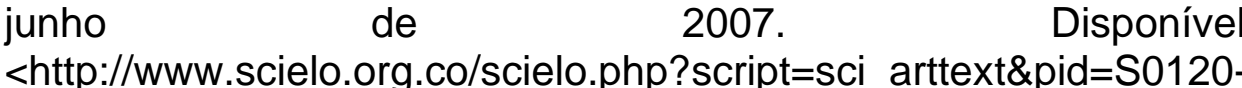

48232007000100004\&lng=en\&nrm=iso>. Acesso em: 30 de julho de 2021.

MARTINS, J. C. G. Sobre revoluções científicas na Matemática. Rio Claro, 2005. 175f. Tese de Doutorado - Instituto de Geociências e Ciências Exatas, Universidade Estadual Paulista, 2005.

OSTERMANN, F.. A Epistemologia de Kuhn. Cad.Cat.Ens.Fis., v.13, n3: p.184196, dez. 1996.

PRODANOV, C. C.; FREITAS, E. C. Metodologia do trabalho científico: métodos e técnicas da pesquisa e do trabalho acadêmico. Novo Hamburgo, RS: Feevale, 2013.

SANTOS, E. E. dos. O infinito de George Cantor: uma revolução paradigmática no desenvolvimento da matemática. 2008. 264p. Tese (doutorado) - Universidade Estadual de Campinas, Instituto de Filosofia e Ciências Humanas, Campinas, SP.

SILVA NETO, S. A.. O que é um paradigma?. Revista de Ciências Humanas (UFSC), v. 45, p. 345-354, 2011. 